\title{
Synthesis of some dipyrrolophenanthroline derivatives as novel macromolecules
}

\author{
Malek Taher Maghsoodlou, ${ }^{*}$ Batool Tahami Pour, Ghasem Marandi, \\ and Niloufar Akbarzadeh \\ Department of Chemistry, The University of Sistan and Balouchestan, P. O. Box 98135-674, \\ Zahedan, Iran \\ E-mail: mt_maghsoodlou@yahoo.com
}

This paper dedicated to Professor Issa Yavari on occasion of his $60^{\text {th }}$ birthday

\begin{abstract}
Some $N$-heterocyclic compounds such as pyrazino[2,3-f][1,10]phenanthroline and dipyrido[3,2$a$ : 2',3'-c]phenazine can react with dialkyl acetylenedicarboxylate to give new helical compounds, which exhibit nonplanarity enforced by the crowding of the pyrrole rings.
\end{abstract}

Keywords: Helical compounds, pyrazino[2,3-f][1,10]phenanthroline, dipyrido[3,2-a:2',3'c]phenazine, dialkyl acetylenedicarboxylate and dipyrrolo phenanthroline

\section{Introduction}

Addition reactions of acetylenic esters to nitrogen-containing heterocycles have been described by several research groups ${ }^{1-9}$ and often lead to various products which may require great experimental skills for successful resolution.

These polycyclic compounds are very interesting molecules not only from theoretical viewpoint (synthesis, reactivity, stereochemistry, etc.) but also for their biological activity. These azapolycyclic compounds show a variety of biological activities (antimicrobial, antineoplastic, antiviral, etc. $)^{10-13}$ and can be effective pharmacophore units of drugs which increase substantially the value of these compounds. For instance some pyrrolo[1,2- $f$ phenanthridines showed unique properties and be able not only to reduce the HIV-induced cytopathogenicity, but also to stimulate the growth of the same MT cells at lower concentrations. ${ }^{14}$

An example of these reactions is the reaction between pyridine and dimethyl acetylenedicarboxylate in methanol, in which the indolizine 1,2,3-tricarboxylate 1 is isolated. ${ }^{15-20}$ 1,10-phenanthroline derivatives also can react with acetylenic esters to give dipyrrolo[1,2- $a: 2^{\prime}, 1^{\prime}-$ 
$k][1,10]$ phenanthroline 2 and pyrrolo[ $1,2-a][1,10]$ phenanthroline derivatives 3 which exhibit nonplanarity enforced by the crowding of the pyrrole rings(Scheme 1). ${ }^{21-26}$
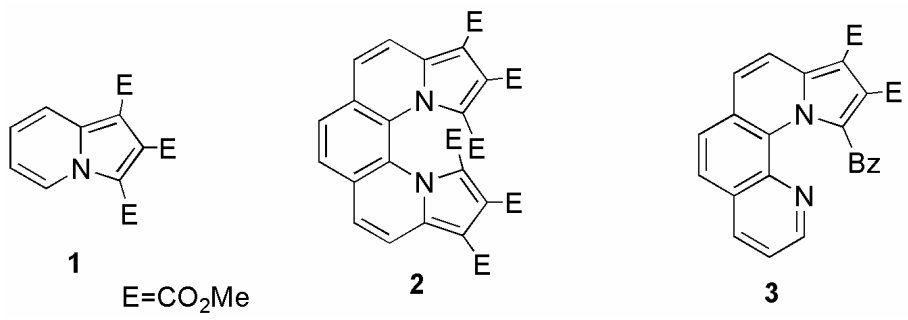

\section{Scheme 1}

The compounds $\mathbf{2}$ and $\mathbf{3}$ are examples of helical compounds. In helical molecules, helicity is inherent in the molecular framework and optical activity ascribed to nonplanarity enforced by the crowding of the pyrrole rings. In order to provide further examples of this remarkable polycyclic compounds we examined the reaction of some phenanthroline derivatives such as pyrazino[2,3$f][1,10]$ phenanthroline (ppl) 4 and dipyrido[3,2-a:2',3'-c]phenazine (dppz) 6 with acetylenic esters giving new helical compounds.

\section{Results and Discussion}

We have found that compounds $\mathbf{4}$ and $\mathbf{6}$ that have structure similar to phenanthroline, can react with dialkyl acetylenedicarboxylates via a one-step reaction in a protic solvent such as methanol to give hexamethyl dipyrrolo[1,2-a:2',1'-o]pyrazino[2,3-f][1,10]phenanthroline $9,10,11,14,15,16$ hexa carboxylate 5 and hexaalkyl diindolizino[6,5- $\left.a: 5^{\prime}, 6^{\prime}-c\right]$ phenazine-11,12,13,16,17,18hexacarboxylate derivatives 7 as new helical compounds in moderate yields (Scheme 2).

The mechanism of cycloaddition is shown in the Scheme 3. The initial addition of $\mathrm{N}$ heterocycle to dialkyl acetylenedicarboxylate and subsequent protonation of the adduct by protic solvent led to the vinyl pyridinium cation which undergoes further reaction with DMAD to produce indolizine (Scheme 3).

Owing to the crowding of the pyrrole rings, it was expected that the skeleton of this heterocyclic system might deviate from planarity, conferring helicity on the molecule. ${ }^{21-22}$ These new compounds are so symmetrical that they exhibit a fairly simple spectrum.

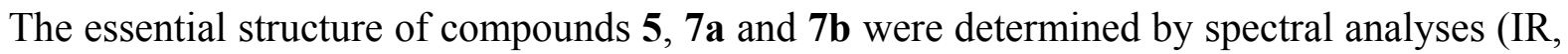
${ }^{1} \mathrm{H}$ NMR, ${ }^{13} \mathrm{C}$ NMR and Mass). In the IR spectrum of these compounds the most important signals are those of the ester groups at $v=1698 \mathrm{~cm}^{-1}$. Conjugation with the heterocyclic ring appears to be a plausible factor in the reduction of the wave numbers of the carbonyl absorption bands. $^{27}$ 

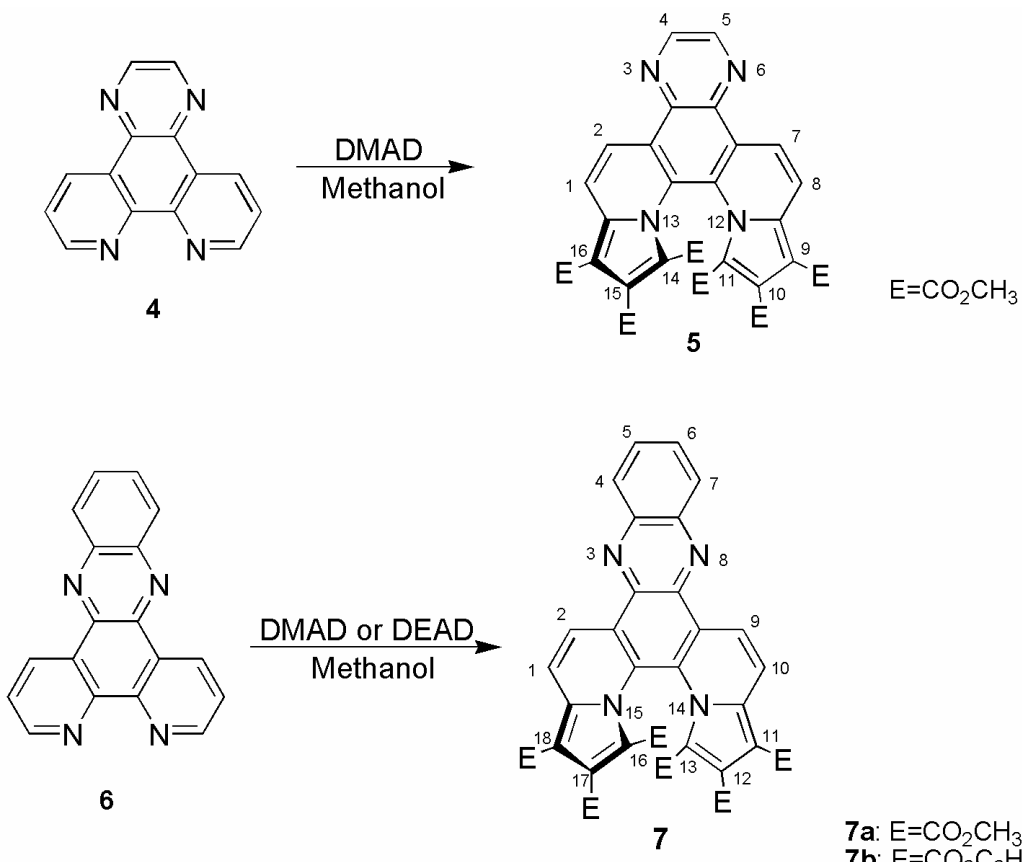

7a: $\mathrm{E}=\mathrm{CO}_{2} \mathrm{CH}_{3}$
7b: $\mathrm{E}=\mathrm{CO}_{2} \mathrm{C}_{2} \mathrm{H}_{5}$

\section{Scheme 2}

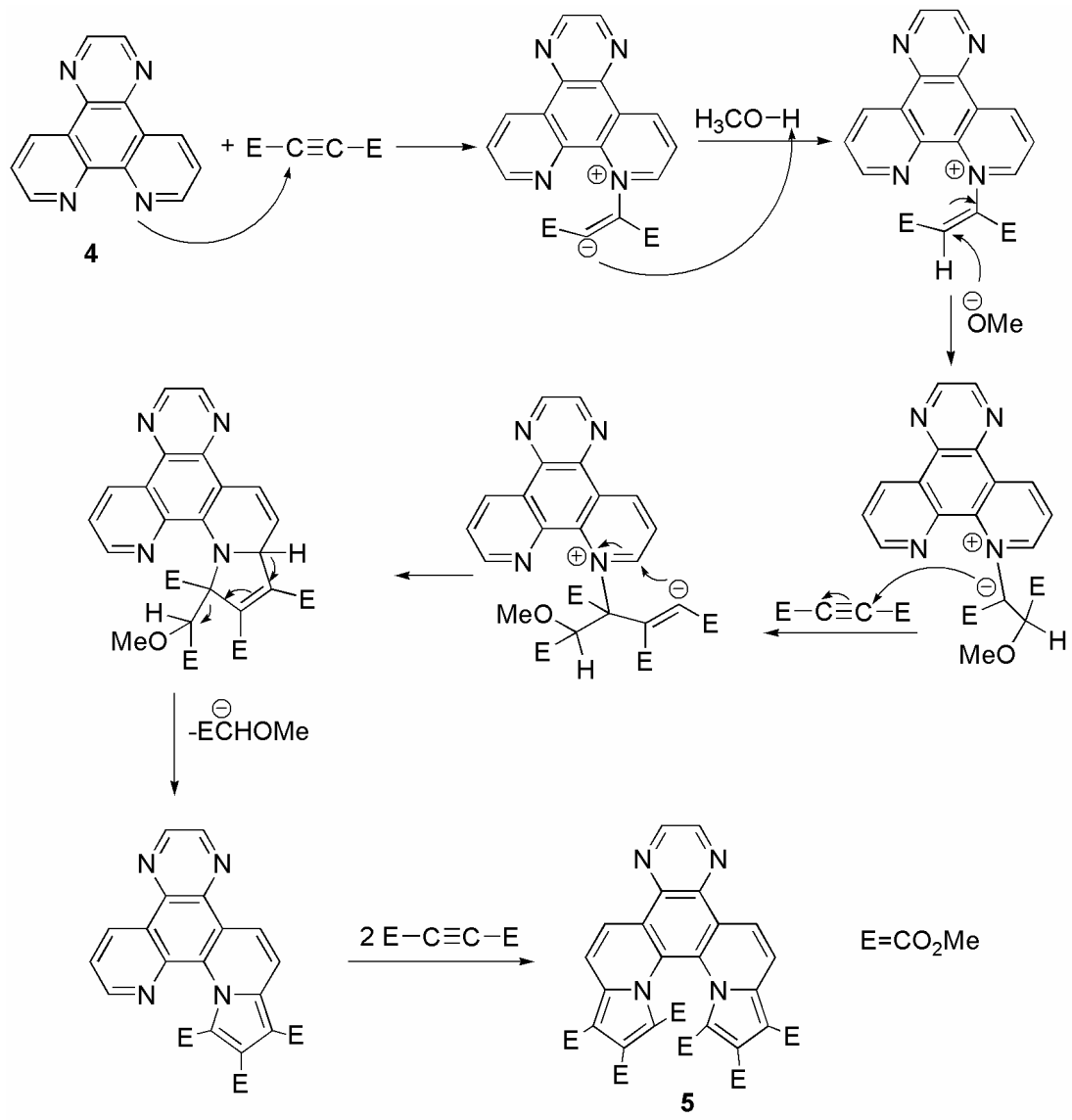

Scheme 3 
The mass spectra of this compound displayed molecular ion peak at appropriate $m / z$ values. Any initial fragmentation involves loss of the ester moieties or ring fragmentation.

The ${ }^{1} \mathrm{H}$ NMR spectrum of 5 recorded in $\mathrm{CDCl}_{3}$ exhibited three single sharp lines at $\delta 3.36$, 4.03, and 4.06 readily recognizable as arising from methoxy groups. The ${ }^{13} \mathrm{C}$ NMR spectrum of 5 displayed three signals for the methoxy groups at $\delta 50.48,51.10,51.77$ and ten signals for the aromatic system nucleus. The chemical shifts of the ester carbonyl groups appear at $\delta 156.30$, 162.19 and 164.47.

The ${ }^{1} \mathrm{H}$ NMR spectrum of compound $7 \mathbf{a}$ for the ester group is analogous to that obtained for 5 but we can see two doublet-doublet peaks at aromatic region that assigned with aromatic system nucleus. In the ${ }^{1} \mathrm{H}$ NMR spectrum of compound $\mathbf{7 b}$ the methylenic hydrogens of the ester group appeared as three $\mathrm{ABX}_{3}$ patterns multiplets that showed that the product is chiral. This behavior can be explained by nonplanarity enforced by the crowding of the pyrrole ring which imparts helical chirality to the molecule. ${ }^{28}$

In conclusion we have demonstrated the synthesis of $\mathbf{5}$ and 7 via a one-step reaction from pyrazino[2,3-f][1,10]phenanthroline and dipyrido[3,2-a:2',3'-c]phenazine. This reaction is a convenient way to obtain azapolycyclic compounds as new helical compounds, which are otherwise very difficult or even impossible to prepare.

\section{Experimental Section}

General Procedures. Melting points were measured on electrothermal 9100 apparatus and are uncorrected. IR spectra were measured on Shimadzu IR-460 spectrometer. ${ }^{1} \mathrm{H}$ and ${ }^{13} \mathrm{C}$ NMR spectra were recorded on Brucker DRX-400 and DRX-300 AVANCE spectrometers at 400.13 and $100.77 \mathrm{MHz}$ for 5 and 300 and $75 \mathrm{MHz}$ for 7, respectively. Mass spectra were recorded on Finnigan-Matt 8430 mass spectrometer operating at an ionization potential of $70 \mathrm{eV}$. Elemental analysis for $\mathrm{C}, \mathrm{H}$ and $\mathrm{N}$ were performed using a Heraeus $\mathrm{CHN}-\mathrm{O}-\mathrm{Rapid}$ analyzer. The compounds 1,10-phenanthroline-5,6-dione, pyrazino[2,3-f][1,10]phenanthroline, dipyrido[3,2$\left.a: 2^{\prime}, 3^{\prime}-c\right]$ phenazine were synthesized according to the literature methods. ${ }^{29-31}$ Dialkyl acetylenedicarboxylates were obtained from Fluka and were used without further purification.

\section{General preparative procedures}

To a magnetically stirred solution of pyrazino[2,3-f][1,10]phenanthroline $(0.11 \mathrm{~g}, 0.5 \mathrm{mmol})$ in methanol $(20 \mathrm{~mL})$ was added dropwise a solution of dimethyl acetylenedicarboxylate $(0.31 \mathrm{~g}, 2.2$ $\mathrm{mmol})$ in methanol $(2 \mathrm{~mL})$ at room temperature over 5 minutes and the mixture refluxed for 24 hours. After this time the resulting brown tar was crystallized from methanol. Elution with petroleum-ether (8:1) gave 5 as pale brown crystals $(0.2 \mathrm{~g}$, yield $62 \%)$, mp: $220-222{ }^{\circ} \mathrm{C}$, IR $(\mathrm{KBr})\left(v_{\max }, \mathrm{cm}^{-1}\right): 1170$ (s, C-O), 1226 (vs, C-O), 1460 (s, C=C), 1515 (m, C=C), 1699 (vs, $\mathrm{C}=\mathrm{O})$ and $1740(\mathrm{~s}, \mathrm{C}=\mathrm{O}) .{ }^{1} \mathrm{H}$ NMR $\left(400 \mathrm{MHz}, \mathrm{CDCl}_{3}\right): \delta_{\mathrm{H}} 3.36,4.03,4.06(18 \mathrm{H}, 3 \mathrm{~s}, 6 \mathrm{OCH} 3)$, $8.9(2 \mathrm{H}, \mathrm{d}, J=6.9 \mathrm{~Hz}, \mathrm{C}-2-\mathrm{H}$ and C-7-H), $9.12(2 \mathrm{H}, \mathrm{s}, \mathrm{C}-4-\mathrm{H}, \mathrm{C}-5-\mathrm{H}), 9.45$ (2H, d, $J=6.9 \mathrm{~Hz}, \mathrm{C}-$ 
1-H and C-8-H). ${ }^{13} \mathrm{C}$ NMR (100 MHz, $\left.\mathrm{CDCl}_{3}\right): \delta_{\mathrm{C}} 50.9$ (q, J=123.5 Hz, $2 \mathrm{OCH}_{3}$ ), 51.1 (q, $J=126.3 \mathrm{~Hz}, 2 \mathrm{OCH}_{3}$ ), 51.8 (q, $J=124.1 \mathrm{~Hz}, 2 \mathrm{OCH}_{3}$ ), 105.7 (s, $2 \mathrm{C}$ ), 117.7 (s, $2 \mathrm{C}$ ), 118.0 (d, $J=163.9 \mathrm{~Hz}, 2 \mathrm{CH}), 121.7$ (s, 2 C), 123.4 (s, 2 C), 125.1 (dd, $J=210.7$ and $J=8.3 \mathrm{~Hz}, 2 \mathrm{CH}$ ), 130.0 (s, 2 C), 137.0 (d, $J=9.3 \mathrm{~Hz}, 2 \mathrm{C}$ ), 137.9 (d, $J=4.3 \mathrm{~Hz}, 2 \mathrm{C}$ ), 144.0 (dd, $J=172.8$ and $J=10.3$ $\mathrm{Hz}, 2 \mathrm{CH}), 156.3,162.25,164.5$ (3s, $6 \mathrm{CO}) . \mathrm{MS}(\mathrm{m} / \mathrm{z}, \%): 656\left(\mathrm{M}^{+}, 3\right), 625$ (2), 597 (3), 553 (7), 538 (7), 507 (10), 494 (3), 449 (4), 421 (7), 363 (10), 332 (7), 304 (24), 152 (13), 59 (100). Anal. Calc for $\mathrm{C}_{32} \mathrm{H}_{24} \mathrm{~N}_{4} \mathrm{O}_{12}$ (656.55): C, 58.54; H, 3.68; N, 8.53\%; Found: C, 58.67; H, 3.44; N, $8.39 \%$.

Hexamethyl diindolizino $\left[6,5-a: 5^{\prime}, 6^{\prime}-c\right]$ phenazine-11, 12, 13, 16, 17, 18-hexacarboxylate (7a). Brown powder, $\left(0.2 \mathrm{~g}\right.$, yield 65\%), mp: $234-236{ }^{\circ} \mathrm{C}$, IR $(\mathrm{KBr})\left(v_{\max }, \mathrm{cm}^{-1}\right): 1176$ (s, C-O), 1225 (vs, C-O), 1433 (s, C=C), $1490(\mathrm{w}, \mathrm{C}=\mathrm{C}), 1703$ (vs, C=O) and 1740 (s, C=O). ${ }^{1} \mathrm{H}$ NMR (300 $\left.\mathrm{MHz}, \mathrm{CDCl}_{3}\right): \delta_{\mathrm{H}} 3.38,4.05,4.08\left(18 \mathrm{H}, 3 \mathrm{~s}, 6 \mathrm{OCH}_{3}\right), 7.96\left(2 \mathrm{H}, \mathrm{dd}, J_{1}=6.3, J_{2}=3.2 \mathrm{~Hz}, \mathrm{C}-5-\mathrm{H}, \mathrm{C}-\right.$ 6-H), 8.42 (2H, dd, $\left.J_{1}=6.3, J_{2}=3.2 \mathrm{~Hz}, \mathrm{C}-4-\mathrm{H}, \mathrm{C}-7-\mathrm{H}\right), 8.92$ (2H, d, J=9.2 Hz, C-2-H, C-9-H), $9.61(2 \mathrm{H}, \mathrm{d}, J=9.2 \mathrm{~Hz}, \mathrm{C}-1-\mathrm{H}, \mathrm{C}-10-\mathrm{H}) .{ }^{13} \mathrm{C} \mathrm{NMR}\left(75 \mathrm{MHz}, \mathrm{CDCl}_{3}\right): \delta_{\mathrm{C}} 51.5$ (q, J=125.8 Hz, 2 $\mathrm{OCH}_{3}$ ), 52.1 (q, $\left.J=123.0 \mathrm{~Hz}, 2 \mathrm{OCH}_{3}\right), 52.8$ (q, $\left.J=124.6 \mathrm{~Hz}, 2 \mathrm{OCH}_{3}\right), 106.5(\mathrm{~s}, 2 \mathrm{C}), 118.4$ (s, 2C), $119.0(\mathrm{~d}, J=164.0 \mathrm{~Hz}, 2 \mathrm{CH}), 123.1(\mathrm{dd}, J=162.5$ and $J=8.0 \mathrm{~Hz}, 2 \mathrm{CH}), 124.7$ (s, 2C), 127.3 (dd, $J=203.1$ and $J=5.8 \mathrm{~Hz}, 2 \mathrm{CH}), 129.6$ (s, 2C), 131.0 (dd, $J=138.4$ and $J=8.7 \mathrm{~Hz}, 2 \mathrm{CH}), 131.1$ (d, $J=10.5 \mathrm{~Hz}, 2 \mathrm{C}), 138.3(\mathrm{dd}, J=8.0$ and $J=0.6 \mathrm{~Hz}, 2 \mathrm{C}), 139.1$ (s, $2 \mathrm{C}), 142.7$ (dd, $J=12.8$ and $J=0.6 \mathrm{~Hz}, 2 \mathrm{C}), 157.28,163.20,165.53$ (3s, $6 \mathrm{CO})$. MS (m/z, \%): $706\left(\mathrm{M}^{+}, 3\right), 675(2), 647(8)$, 603 (8), 588 (48), 557 (8), 544 (7), 471 (7), 413 (9), 354 (18), 294 (10), 207 (13), 198 (6), 177 (39), 59 (43). Anal. Calc for $\mathrm{C}_{36} \mathrm{H}_{26} \mathrm{~N}_{4} \mathrm{O}_{12}$ (706.61): C, 61.19; H, 3.71; N, 7.93\%; Found: C, $61.23 ; \mathrm{H}, 3.82 ; \mathrm{N}, 7.78 \%$.

Hexaethyl diindolizino $\left[6,5-a: 5^{\prime}, 6^{\prime}-c\right]$ phenazine 11, 12, 13, 16, 17, 18 hexacarboxylate (7b). Brown powder, $\left(0.25 \mathrm{~g}\right.$, yields 65\%), mp: $223-225{ }^{\circ} \mathrm{C}$; IR $(\mathrm{KBr})\left(v_{\max }, \mathrm{cm}^{-1}\right): 1175$ (vs, C-O), 1205 (vs, C-O), 1460 (s, C=C), 1497 (m, C=C), 1700 (vs, C=O) and 1715 (s,C=O). ${ }^{1} \mathrm{H}$ NMR $\left(300 \mathrm{MHz}, \mathrm{CDCl}_{3}\right): \delta_{\mathrm{H}} 1.20\left(6 \mathrm{H}, \mathrm{t}, J=7.2 \mathrm{~Hz}, 2 \mathrm{CH}_{3}\right), 1.46-1.53\left(12 \mathrm{H}, \mathrm{m}, 4 \mathrm{CH}_{3}\right), 3.77(4 \mathrm{H}, \mathrm{q}$, $\left.J=7.2 \mathrm{~Hz}, 2 \mathrm{CH}_{2}\right), 4.45-4.55\left(8 \mathrm{H}, \mathrm{m}, J=7.2 \mathrm{~Hz}, 4 \mathrm{CH}_{2}\right), 7.94\left(2 \mathrm{H}, \mathrm{dd}, J_{1}=6.4, J_{2}=3.2 \mathrm{~Hz}, \mathrm{C}-5-\mathrm{H}\right.$, C-6-H), 8.41(2H, dd, $\left.J_{1}=6.4, J_{2}=3.2 \mathrm{~Hz}, \mathrm{C}-4-\mathrm{H}, \mathrm{C}-7-\mathrm{H}\right), 8.93(2 \mathrm{H}, \mathrm{d}, J=9.2 \mathrm{~Hz}, \mathrm{C}-2-\mathrm{H}, \mathrm{C}-9-\mathrm{H})$, $9.58(2 \mathrm{H}, \mathrm{d}, J=9.2 \mathrm{~Hz}, \mathrm{C}-1-\mathrm{H}, \mathrm{C}-10-\mathrm{H}) .{ }^{13} \mathrm{C} \mathrm{NMR}\left(75 \mathrm{MHz}, \mathrm{CDCl}_{3}\right): \delta_{\mathrm{C}} 13.8$ (q, J=82.3 Hz, 2 $\mathrm{OCH}_{2} \mathrm{CH}_{3}$ ), 14.1 (q, $J=95.6 \mathrm{~Hz}, 2 \mathrm{OCH}_{2} \mathrm{CH}_{3}$ ), 14.4 (q, $J=84.6 \mathrm{~Hz}, 2 \mathrm{OCH}_{2} \mathrm{CH}_{3}$ ), 60.8 (br, 2 $\mathrm{OCH}_{2} \mathrm{CH}_{3}$ ), 60.8 (br, $2 \mathrm{OCH}_{2} \mathrm{CH}_{3}$ ), 61.7 (br, $2 \mathrm{OCH}_{2} \mathrm{CH}_{3}$ ), 106.6 (s, 2C), 118.5 (s, $2 \mathrm{C}$ ), 119.0 (d, $J=162.9 \mathrm{~Hz}, 2 \mathrm{CH}), 122.9$ (s, $2 \mathrm{C}), 124.4$ (dd, $J=124.2$ and $J=8.2 \mathrm{~Hz}, 2 \mathrm{CH}), 127.5$ (dd, $J=9.8$ and $J=0.8 \mathrm{~Hz}, 2 \mathrm{C}), 129.6(\mathrm{~d}, J=151.5 \mathrm{~Hz}, 2 \mathrm{CH}), 130.9$ (dd, $J=151.7$ and $J=10.0 \mathrm{~Hz}, 2 \mathrm{CH}$ ), 131.1 (s, 2 C), 138.5 (dd, $J=7.0$ and $J=0.6 \mathrm{~Hz}, 2 \mathrm{C}$ ), 139.3 (d, $J=0.6 \mathrm{~Hz}, 2 \mathrm{C}), 142.6$ (d, $J=0.8 \mathrm{~Hz}$, 2 C), 157.3, 162.9, 165.0 (3s, 6 CO). MS (m/z, \%): $791\left(\mathrm{M}^{+}, 1\right), 746$ (1), $718(2), 674$ (3), 644 (24), 599 (34), 497 (7), 444 (19), 409 (17), 381 (22), 355 (37), 354 (70), 353 (40), 341 (4), 249 (8), 178 (8), 177 (6). Anal. Calc for $\mathrm{C}_{42} \mathrm{H}_{38} \mathrm{~N}_{4} \mathrm{O}_{12}$ (790.77): C, 63.79; H, 4.84; N, 7.09\%; Found: C, 63.86; H, 4.97; N, 6.94\%. 


\section{Acknowledgements}

We gratefully acknowledge financial support from the Research Council of University of Sistan and Balouchestan.

\section{References}

1. (a) Acheson, R. M. Adv. Heterocycl. Chem. 1963, 1, 125. (b) Acheson, R. M.; Elmore, N. F. Adv. Heterocycl. Chem. 1978, 23, 263.

2. Demoulin, A.; Gorissen, H.; Hesbain-Frisque, A. M.; Ghosez, L. J. Am .Chem. Soc. 1975, 97, 4409 .

3. Aue, D. H.; Thomas, D. J. Org. Chem. 1975, 40, 2360.

4. Crabtree, A.; Johnson, A. W. J. Chem. Soc. 1962, 1510.

5. (a) Acheson, R. M.; Foxton, M. W.; Abbott, P. J.; Mills, K. R. J. Chem. Soc. C. 1967, 882.

(b) Acheson, R. M.; Abbott, P. J.; Foxton, M. W.; Raulins, N. R.; Robinson, G. E. J. Chem. Soc. Perkin Trans. 1972, 1, 2182.

6. Diels, O.; Alder, K.; Winckler, H.; Petersen, E. Liebigs Ann. Chem. 1932, 498, 1.

7. Heaney, F.; McArdle, P. ARKIVOC 2003, (vii), 161.

8. Wahe, H.; Mbafor, J. T.; Cherkasov, R. A. ARKIVOC 2003, (xv), 170.

9. Katritzky, A. R.; Rogers, J. W. ARKIVOC 2004, (viii), 52.

10. Ungureanu, M.; Mangalagiu, I.; Grosu, G.; Petrovanu. M. Ann. Pharmaceutiques Francaise 1997, 55, 69.

11. Lednicer, Mitscher, L. A.; Georg, G. The Organic Chemistry of Drug Synthesis 1990, 4, 112.

12. Feigon, J.; Denny, W. A.; Leupin, W.; Kearns, D. R. J. Med. Chem. 1984, 27, 450.

13. Hertzberg, R. P.; Sammes, P.G. In: Comprehensive Medicinal Chemistry 1990, 2, 753.

14. Almerico, A. M.; Mingoia, F.; Diana, P.; Barraja, P.; Montalbano, A.; Lauria, A. Eur. J. Med. Chem. 2002, 37, 3.

15. Acheson, R. M.; Woollard, J. M. J. Chem. Soc. C. 1971, 3296.

16. Acheson, R. M.; Talor, G. A. J. Chem. Soc. 1960, 1691.

17. Crabtree, A.; Johnson, A. W.; Tebby, J. C. J. Chem. Soc. 1961, 3497.

18. Flitsch, W. In: Comprehensive Heterocyclic Chemistry; Katritzky, A. R.; Rees, C. W. Pergamon: London, 1984; 3, pp 443.

19. Swinbourne, F. J.; Hunt, H. J.; Klinkert, G. Adv. Heterocycl. Chem. 1987, 23, 103.

20. Katritzky, A. R.; Qiu, G.; Yang, B. J. Org. Chem. 1999, 64, 7618.

21. Yavari, I.; Maghsoodlou, M. T.; Pourmossavi, A. J. Chem. Research (S) 1997, 213.

22. Dumitrascu, F.; Mitan, C. I.; Draghici, C. Tetrahedron Letter 2001, 42, 8379.

23. Dumitrascu, F.; Caira, M. R.; Draghici, C.; Caproiu, M. T. Molecules 2005, 10, 321. 
24. Dumitrascu, F.; Mitan, C. I.; Draghici, C.; Caproiu, M. T. Rev. Roum. Chim. 2002, 47, 881; Chem. Abstr. 2004, 140, $111296 \mathrm{e}$.

25. Dumitrascu, F.; Mitan, C. I.; Draghici, C.; Caproiu, M. T.; Raileanu, D. Rev. Roum. Chim. 2002, 53, 787; Chem. Abstr. 2003, 138, 368790m.

26. Ramona, D.; Rotaru, A.; Drochioiu, G.; Druta, I. J. Heterocycl. Chem. 2003, 40, 283.

27. Silverstein, R. M.; Bassler, G. C.; Morril, T. C. In: Spectrometric Identification of Organic Compounds; Wiley: New York, $5^{\text {th }}$ Edn., 1991, pp 111.

28. Eliel, E. L.; Wilen, S. H. In: Stereochemistry of Organic Compounds; Wiley: New York, 1994, pp 1163.

29. Amouyal, E.; Homsi, A. J. Chem. Soc. Dalton Trans. 1990, 1841.

30. Delgadillo, A.; Romo, P.; Leiva, A. M.; Loeb, B. Helv. Chem. Acta 2003, 86, 2110.

31. Dickeson, J. E.; Summers, L. A. Aust. J. Chem. 1970, 23, 1023. 